\title{
Cellular prion protein accelerates colorectal cancer metastasis via the Fyn-SP1-SATB1 axis
}

\author{
QIANWEI WANG, JIANMING QIAN, FANGRUI WANG and ZHENYU MA \\ Department of Surgery, Huashan Hospital, Fudan University, Shanghai 200032, P.R. China
}

Received April 23, 2012; Accepted June 8, 2012

DOI: $10.3892 /$ or.2012.2025

\begin{abstract}
The cellular prion protein ( $\mathrm{PrPc})$ is a glycoprotein anchored by glycosylphosphatidylinositol to the cell surface and is abundantly expressed in various tissues. The putative roles of PrPc are thought to be related to cell signaling, survival, and differentiation and cancer progression. In this study, we demonstrated that the expression of PrPc correlates with a more aggressive and histologically unfavorable disease in colorectal carcinomas. Moreover, we found that PrPc mediates the process of epithelial-mesenchymal transition and, thereby, promotes CRC metastasis. Transcriptome profiling of PrPc-depleted cells revealed downregulation of the special AT-rich sequence-binding protein-1 (SATB1). PrPc is demonstrated to be involved in regulating SATB1 expression via the Fyn-SP1 pathway. Since SATB1 has been previously proposed as a key protein that controls tumor development and progression, knockdown of PrPc resulted in a reduced metastatic capacity in CRC cells, as well as a reduction in distant metastases in vivo. In conclusion, our data characterize a novel molecular mechanism that links PrPc expression to the regulation of CRC metastasis. Targeting PrPc will, therefore, be a promising strategy to overcome the metastatic advantage in colorectal tumors.
\end{abstract}

\section{Introduction}

Colorectal carcinoma (CRC) is one of the most commonly diagnosed cancers which remains a leading cause of cancerrelated death and $\sim 50 \%$ of patients with colorectal cancer develop synchronous or metachronous liver metastases (1). The 5 -year survival rate of colorectal cancer patients with metastatic disease is $<10 \%$ (2). Understanding the biological mechanism of metastasis is important for development of new treatment strategies and markers predictive of metastasis. Several recent studies have implied that targeted therapies in conjunction with chemo- or radiotherapy is a potential approach for a rational molecular-based tumor therapy in colorectal cancer $(3,4)$.

Correspondence to: Dr Zhenyu Ma, Department of Surgery, Huashan Hospital, Fudan University, Shanghai 200032, P.R. China E-mail: will_lqq@hotmail.com

Key words: cellular prion protein, metastasis, colorectal cancer, epithelial-mesenchymal transition, sequence-binding protein-1
PrPc is a ubiquitous glycoprotein highly expressed in neurons, mostly anchored at the cell surface via a C-terminal glycosylphosphatidylinositol (GPI) moiety. The best described property of $\mathrm{PrPc}$ is its high affinity for $\mathrm{Cu}(\mathrm{II})$, which suggests that PrPc could participate in copper metabolism and protection against oxidative stress (5). Indeed, primary cultures of neurons from $\mathrm{PrP}^{-/-}$mice exhibit increased susceptibility to oxidative stress (6). Also, PrPc has been proposed to be involved in neurite outgrowth and neuronal survival in cell cultures of primary neurons (7).

Recently, emerging evidence have indicated that cellular prion protein $(\mathrm{PrPc})$ may be implicated in tumor cell biology (8-11). PrPc overexpression is correlated to the acquisition by tumor cells of a phenotype for resistance to cell death induced by antitumor drugs. Upon temozolomide treatment in gliomas, PrPc exerts its antiapoptotic activity by inhibiting PKA-mediated par-4 phosphorylation at the T155 residue that are important for par-4 activation, nuclear entry and initiation of apoptosis (12). Also, PrPc significantly promoted the adhesive, invasive, and in vivo metastatic capacities of the gastric cancer cells (11). Moreover, overexpression of PrPc accelerated the proliferation of gastric cancer cells through transcriptional activation of cyclin D3 to faciliate G1/S-phase transition (13). Therefore, PrPc seems to serve as a promising target for novel anti-cancer therapies.

We demonstrate that PrPc expression is associated with metastatic potential of colorectal cancer cells since it mediates invasive and metastatic capacities by regulating SATB1 expression via epigenetic activation of Fyn-SP1 pathway. PrPc depletion inhibits tumor metastasis of colorectal cancer both in vivo and in vitro. Based on above, these studies suggest PrPc as a promising therapeutic target against advanced metastatic human CRCs.

\section{Materials and methods}

Cell lines. The human colorectal carcinoma cell line SW480 was purchased from American Type Culture Collection (Manassas, VA, USA). LIM 2405 cells, which derive from a poorly differentiated primary human colon adenocarcinoma, were obtained from Dr R. Whitehead (Ludwig Institute, Melbourne, Australia). Cells were maintained in growth medium at $37^{\circ} \mathrm{C}$ in a humidified incubator.

Immunoblotting. Total protein was extracted from cells using RIPA lysis buffer (Santa Cruz Biotechnology, Santa Cruz, 
CA, USA). Protein extract (50 $\mu \mathrm{g} / \mathrm{lane})$ was electrophoresed, transferred to PVDF membranes, and incubated overnight with primary antibodies against PrPc, E-cadherin, N-cadherin (all Santa Cruz Biotechnology), SATB1 and $\beta$-actin (both SigmaAldrich, St. Louis, MO, USA). Membranes were then treated with the appropriate HRP-conjugated secondary antibodies (Invitrogen, Carlsbad, CA, USA). Detection was carried out using the reagents provided in the ECL Plus kit (GE Healthcare, Milwaukee, WI, USA).

Quantitative real-time PCR. qPCR was performed using TaqMan probes from Applied Biosystems, according to the manufacturer's instructions. Reactions were carried out in an ABI 7000 sequence detector (Perkin-Elmer) and results were expressed as fold change calculated by the $\Delta \Delta \mathrm{Ct}$ method relative to the control sample or to the first sample quantified. GAPDH or $\beta$-actin was used as internal normalization controls.

In vitro migration and invasion assays. A bioassay for in vitro cell migration/invasion using Matrigel Invasion Chambers (Corning Costar, Corning, NY, USA) was performed as described previously (14).

Chromatin immunoprecipitation. LIM2405 cells were transfected with PrPc siRNA for $72 \mathrm{~h}$ prior to carrying out ChIP using antibodies against SP1. A total of 1.0-2.5\% of each immunoprecipitation was assayed by PCR using primers specific for a region of interest.

Luciferase reporter assays. Cells in 24-well plates were transiently transfected with different $S A T B 1$ promoter reporter constructs and pRLTK Renilla luciferase plasmid (Promega) using FuGENE 6 (Roche). Cell extracts were prepared $48 \mathrm{~h}$ after transfection, and luciferase activity was measured using the dual-luciferase reporter assay system (Promega).

Immunofluorescence microscopy analysis. Immunofluorescence microscopy analysis using antibodies against $\beta$-catenin (Sigma) was carried out as described previously (14).

In vitro kinase assays. For studies of Fyn activation in the presence and absence of $\mathrm{PrPc}$, in vitro kinase assays were carried out on Fyn, as previously described (15).

Animal studies. Indicated tumor cells were orthotopically inoculated into the serosa of the intestine after the laparotomy. The growth and metastasis of the tumors were monitored by weekly bioluminescence imaging using intravital imaging system (Nikon). End-point assays were conducted 7 weeks after the inoculation unless animal sacrifice was required because of significant morbidity. The animal welfare guidelines of the Huashan Hospital's Research Institute Institutional Animal Use and Care Committee were followed and the experimental protocol was approved by the committee.

Patient study. Patients were identified from the HuaShan Hospital records. The ethics committees of the participating hospital approved the written informed consent for the collection of tumor tissue obtained from each patient. Histological classification of colorectal carcinomas proposed by the World Health
Organization was adopted. Three-micrometer sections were cut from paraffin blocks onto silanized slides, and the sections were immunostained using antibodies against PrPc (Sigma), E-cadherin (BioGenex, San Ramon, CA, USA), and vimentin (Sigma) respectively.

Statistical analyses. Statistical analyses were calculated by SPSS software (SPSS, Chicago, IL, USA). The results are presented as the mean \pm SEM. ANOVA, Student's t-test analysis, and Dunnett's multiple comparison tests were used to compare mean values. $\mathrm{P}<0.05$ was consiedered statistically significant.

\section{Results}

PrPc expression in colorectal carcinoma tissues. Histological analysis revealed that PrPc, primarily located at the membrane/ cytoplasm, were significantly increased in tumor tissues compared with those in adjacent normal mucosal tissues (Fig. 1A). The Pearson's $\chi^{2}$ test indicated that PrPc expression was closely associated with tumor encapsulation, microvascular invasion, histological grade and TNM stage. Furthermore, we found that the expression of PrPc did not correlate with other clinicopathological characteristics such as age, gender or tumor diameter (Fig. 1B). Interestingly, all samples from CRC patients reproducibly demonstrated that PrPc-positive cells were sporadically distributed within the tumor parenchyma, but were arranged in dense clusters at the invasive front of the tumor. PrPc-positive cells were often found in more differentiated epithelial cells but were essentially negative for the epithelial marker cytokeratin and positive for the mesenchymal marker vimentin (Fig. 1C).

$\operatorname{PrPc}$ promotes metastatic potential of $C R C$ cells. It has been reported that EMT-like cell dedifferentiation takes place at the invasive front of CRCs (16). Combined with the fact that higher expression of PrPc at the invasive front was noted in CRC tissues, we hypothesized that PrPc might be associated with the epithelial-mesenchymal transition (EMT). To confirm the relationship between them, we investigated the effects of PrPc expression on the phenotypic changes of CRC cells. We observed that while SW480 cells grew as tightly packed colonies characteristic of epithelial cells, cells treated with pCDNA3.0-PRNP appeared actively spreading and had lost the majority of their cell-cell contacts (Fig. 2A). These phenomena were associated with decreased expression of E-cadherin, up-regulation of $\mathrm{N}$-cadherin and translocation of $\beta$-catenin from membrane to nucleus (Fig. 2B and C). Conversely, mesenchymal-like LIM2405 cells transfected with PrPcsiRNA obtained a characteristic epithelial porphology (Fig. 2A) and displayed downregulated N-cadherin, upregulated E-cadherin and cytoplasmic restoration of nuclear $\beta$-catenin (Fig. 2B and C). Thus, it can be concluded that PrPc induces EMT in epithelial CRC cells.

EMT contributes to invasive and metastatic tumor growth. In vitro transwell migration assays showed that PrPc promoted the migration of SW480 cells, while inhibition of PrPc reduced the motility of LIM2405 cells (Fig. 2D). We also orthotopically implanted GFP-expressing and shPRNP-treated cells in SCID mice. Although no significant growth-inhibitory effect was observed in the treated groups as compared with the control groups, LIM2405 cells formed a greater number of 
A

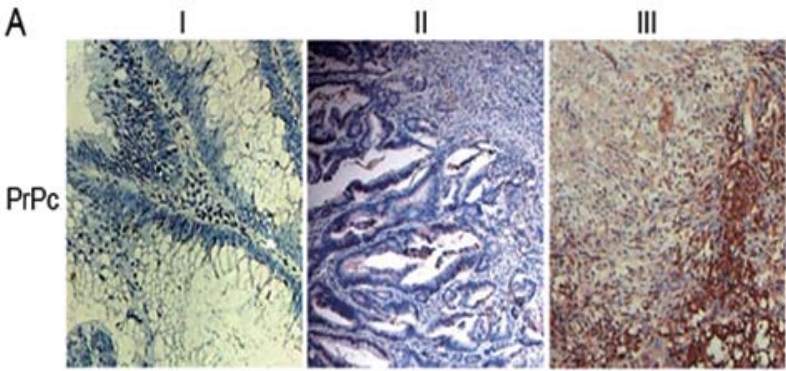

C

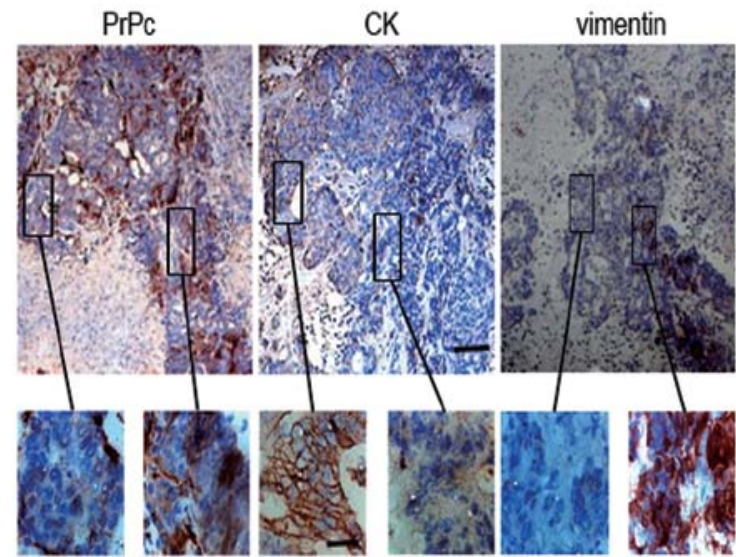

B

\begin{tabular}{|c|c|c|c|}
\hline \multirow[t]{2}{*}{ variables } & \multicolumn{2}{|c|}{ PrPc staining } & \multirow[b]{2}{*}{$P$} \\
\hline & $\begin{array}{l}\text { high } \\
(n=87)\end{array}$ & $\begin{array}{c}\text { low } \\
(n=62)\end{array}$ & \\
\hline \multicolumn{4}{|l|}{ Age } \\
\hline$<50$ & 23 & 17 & \\
\hline$>50$ & 64 & 49 & 0.107 \\
\hline \multicolumn{4}{|l|}{ Gender } \\
\hline male & 6 & 5 & \\
\hline female & 81 & 59 & 0.232 \\
\hline \multicolumn{4}{|c|}{ Tumor Diameter (cm) } \\
\hline$<5$ & 37 & 29 & \\
\hline$>5$ & 50 & 33 & 0.158 \\
\hline \multicolumn{4}{|c|}{ Tumor encapsulation } \\
\hline complete & 31 & 36 & \\
\hline imcomplete & 56 & 26 & $<0.05$ \\
\hline \multicolumn{4}{|c|}{ Microvascular invasion } \\
\hline no & 18 & 34 & \\
\hline yes & 69 & 28 & $<0.01$ \\
\hline \multicolumn{4}{|c|}{ Histological grade } \\
\hline |III & 36 & 43 & \\
\hline$\| I V$ & 51 & 19 & $<0.01$ \\
\hline \multicolumn{4}{|l|}{ TMN stage } \\
\hline । & 31 & 45 & \\
\hline$\|$ UII & 56 & 17 & $<0.01$ \\
\hline
\end{tabular}

Figure 1. PrPc correlates with metastatic potential of human colorectal carcinomas. (A) Representative immunostaining of PrPc in colorectal tissues with different histological grades. (B) Correlation between PrPc staining and clinicopathological characteristics in 203 CRC patients. (C) A representative case of serial sections from a CRC patient. CRC cells locating at the invasive front express higher level of PrPc and vimentin, but relatively lower level of CK. Scale bars, $20 \mu \mathrm{m}$.

A
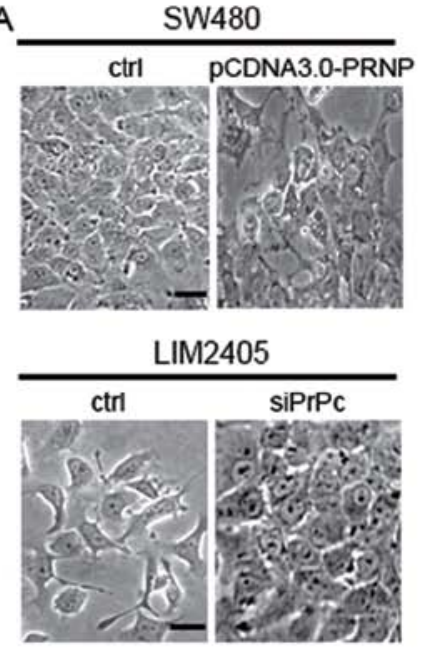

D

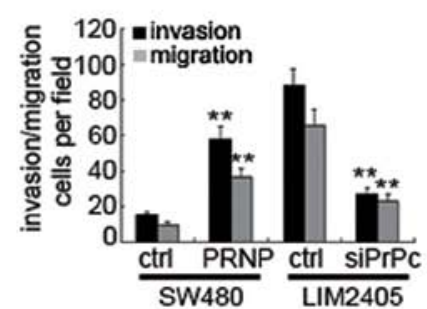

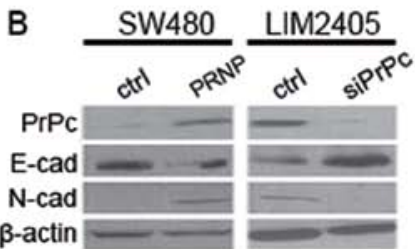
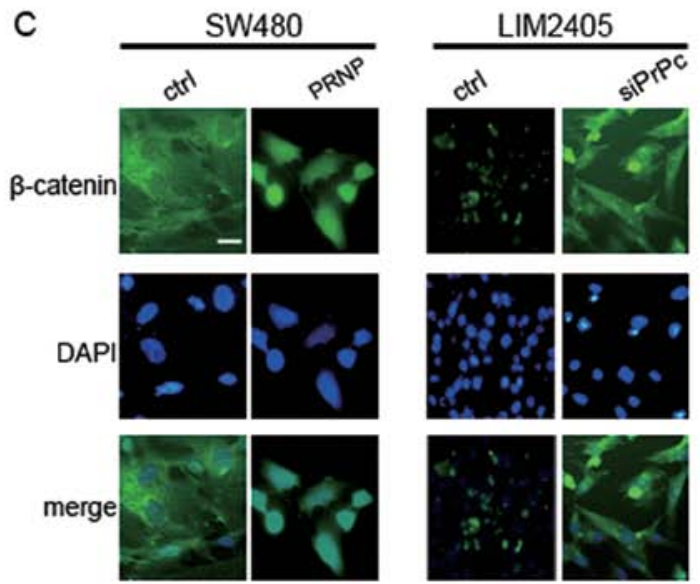

$E$

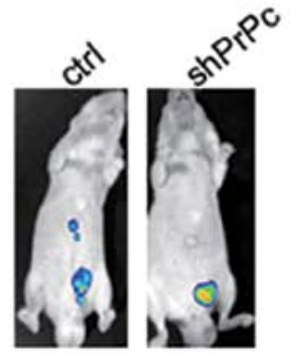

$\mathrm{F}$

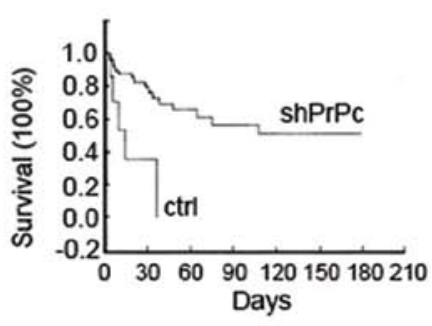

Figure 2. Effects of PrPc on metastatic capability of CRC cell lines. After SW480 and LIM2405 cells were transfected with pCDNA3.0-PRNP or siPrPc, respectively. EMT was examined by phase-contrast photomicrographs (A), immunoblotting of $\mathrm{PrPc}$, E-cadherin and $\mathrm{N}$-cadherin (B), immunofluorescence of $\beta$-catenin and the 2-chamber transwell assays (C) were performed. (D) Representative images of in vivo metastases of LIM2405 cells treated with or without shPrPc are shown through fluorescence of the tumor cells. (E) Cumulative survival rate of nude mice implanted orthotopically with the 2 cell types. The survival curve was plotted according to the method of Kaplan-Meier $(\mathrm{n}=10) .{ }^{* *} \mathrm{P}<0.05$ versus control cells. Scale bars, $20 \mu \mathrm{m}$. 
A

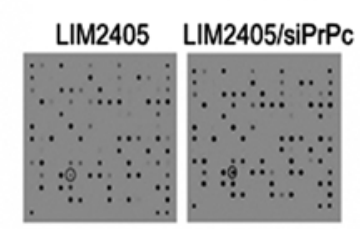

$\mathrm{E}$

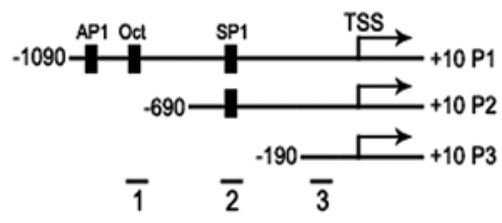

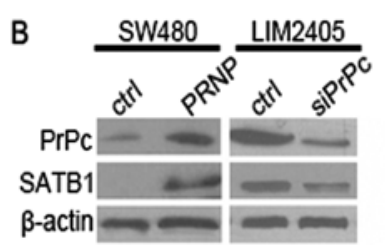

F

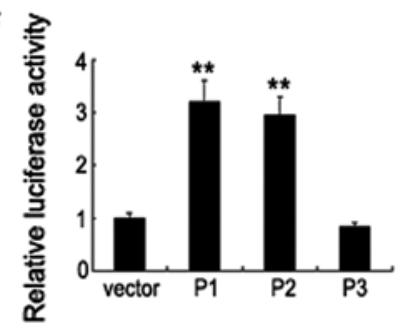

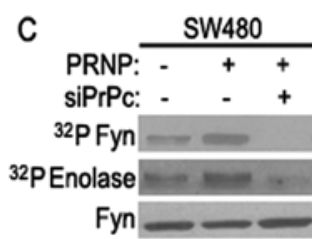

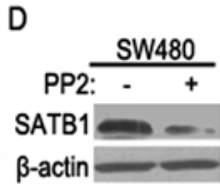

G

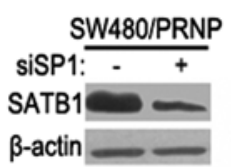

$\mathrm{H}$

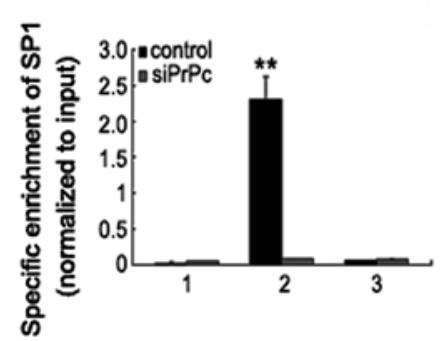

Figure 3. PrPc regulates SATB1 expression in a Fyn-SP1-dependent manner. (A) Array hybridization analysis of gene expression extracted from LIM2405 cells treated without (upper array) or with (lower array) PrPc siRNA. (B) PrPc and SATB1 expressions in PrPc-overexpressed SW480 and PrPc-depleted LIM2405 cells were assessed by immunoblotting, respectively. After PrPc-overexpressed SW480 cells were treated with siPrPc or PP2, Fyn (C) or SATB1 (D) expression were determined by immunoblotting. (E) Scheme of SATB1 luciferase reporter constructs. (F) LIM2405 cells were transfected with the indicated constructs for $36 \mathrm{~h}$, after which luciferase analysis was performed. (G) After PrPc-overexpressed SW480 cells were treated with siSP1, SATB1 expression were assessed by immunoblotting. (H) Cells were treated with or without siPrPc for $24 \mathrm{~h}$ before ChIP analysis using SP1 antibody. Genomic DNA fragments covering region surrounding the SATB1 TSS as indicated in (E) were analyzed by real-time PCR. Enrichment was normalized to input control.

metastases than LIM2405-shRNA cells in the liver (Fig. 2E). Seventeen weeks after treatment, the Kaplan-Meier plot assessment showed a significant prolongation of surviving mice bearing shPRNP-treated colorectal tumors (Fig. 2F). These experiments confirmed the role of PrPc in the promotion of CRC metastasis.

$P r P c$ regulates SATB1 expression via Fyn-SPI pathway. To find genes involved in the observed PrPc-mediated CRC metastasis, we performed transcriptome profiling. After $72 \mathrm{~h}$ of PrPc depletion in LIM2405 cells, analysis of array hybridization revealed 89 differentially expressed genes. SATB1 was further investigated because it was the most significantly changed metastasis-related gene in PrPc-depleted cells (Fig. 3A). As shown in Fig. 3B, SATB1 was downregulated in LIM2405 cells $72 \mathrm{~h}$ after PrPc silencing, according with the results obtained from immunoblot analysis. On the contrary, SATB1 expression was significantly enhanced upon pCDNA3.0-PrPc transfection into SW480 cells in which PrPc is negligibly expressed.

Among the potential signaling pathways which are modulated by PrPc (PI3K/Akt, cAMP/PKA, PKC, Fyn, and Erk1/2), only Fyn pathway is constitutively activated in PrPc-overexpressing SW480 cells, which could not be observed after PrPc siRNA treatment (Fig. 3C). SATB1 expression was primarily abolished by exposure of the cells to PP2 (a specific Fyn inhibitor), even in the presence of PrPc (Fig. 3D). To further determine which response element participates in the regulation of SATB1 promoter activity in response to $\mathrm{PrPc}$, we constructed a series of SATB1 promoter reporter mutation vectors (P1 to P3). Using MatInspector software, an algorithmic prediction of potential transfactor binding sites identified putative-binding sites for AP1, Oct and SP1 (Fig. 3E). P1 to P2 were significantly activated in LIM2405 cells. Deletion of the putative SP1 site in the SATB1 promoter (P3) strongly diminished the SATB1 promoter activity (Fig. 3F). Moreover, PrPc-mediated SATB1 expression was primarily abrogated by treatment with SP1siRNA in
A
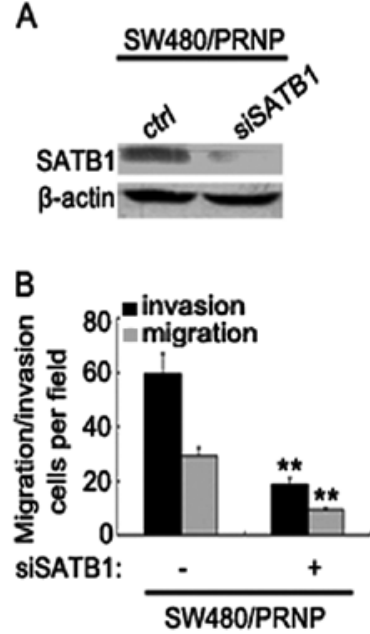

D

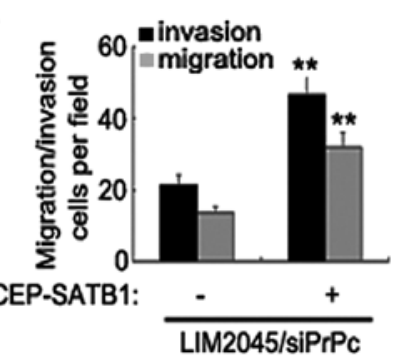

Figure 4. PrPc-Fyn-SATB1 axis plays a critical role in metastasis of colorectal cancer cells. After siSATB1 or pCEP4-SATB1 was transfected into PrPc-overexpressed SW480 or PrPc-depleted LIM2405 cells, respectively, immunoblotting of SATB1 (A and B) and the 2-chamber transwell assays $(\mathrm{C}$ and $\mathrm{D})$ were performed.

PrPc-overexpressed cells, suggesting that the PrPc-SP1 axis is required for SATB1 expession (Fig. 3G). In agreement with this, a chromatin immunoprecipitation (ChIP) assay revealed that the promoter region containing the putative SP1 site was specifically co-immunoprecipitated by anti-SP1 antibody, whereas PrPc knockdown significantly attenuated this effect (Fig. 3H). Thus, these studies demonstrate that the Fyn-SP1 signaling pathway plays a critical role in the PrPc-mediated upregulation of SATB1.

PrPc-SATB1 axis is essential for CRC metastasis. To evaluate whether SATB1 mediates the increase of CRC metastasis by PrPc, we used siRNAs to specifically block PrPc-induced SATB1 
expression (Fig. 4A). Knockdown of SATB1 resulted in drastic reduction of invasive and metastatic capacity of LIM2405 cells (Fig. 4B). We also overexpressed SATB1 in a PrPc knockdown clone of LIM2405 cells (Fig. 4C). PrPc-depleted cells exhibited increased metastatic capacities upon ectopic SATB1 expression, whereas the untreated cells yielded lower metastasis (Fig. 4D). These results provide direct evidence that SATB1 plays an essential role in regulating CRCs metastasis by PrPc.

\section{Discussion}

PrPc has emerged as an important molecule to modulate cancer cell proliferation $(13)$, drug resistance $(12,17)$ and metastasis (11). Our study used an extensive collection of CRC samples to show that PrPc expression was clearly associated with the differentiation and TNM stage of CRCs, which were similar to previous reports in other malignancies such as breast cancer (9) and gastric cancer (12). Indeed, several pieces of evidence in this study showed a close association between PrPc expression and CRC metastasis. First, we observed that PrPc was specifically expressed at the invasive front of CRCs, where carcinoma cells gain the characteristics of EMT and facilitate tumor invasion. Second, functional assays showed that ectopic $\mathrm{PrPc}$ expression promoted in vitro metastatic potential of CRC cells, whereas inhibition of PrPc significantly reduced the motility of cancer cells. Consistently, using an orthotopic xenograft model, we found that knockdown of PrPc in the implanted CRC cells remarkeably abolished the number of distant metastases. These results lend further credence to the notion that PrPc plays a crucial role in regulating CRC progression and metastasis.

The mechanisms for the functional role of PrPc in promoting tumor malignancy still remain poorly understood. The present results characterized a novel PrPc-dependent pathway, where PrPc accelerated tumor metastasis by upregulating SATB1 expression. SATB1 is a matrix attachment region (MARAR)binding protein that participates in higher-order chromatin organization and tissue-specific gene expression. SATB1 has recently been shown to be the 'master regulator' of global gene expression $(18,19)$. SATB1 markedly altered the gene expression profile of cancer cells to induce an aggressive phenotype that promotes tumor metastasis. Strikingly, depletion of SATB1 results in reduced cancer progression and the reversion of metastatic cells to normal appearance (18). Therefore, a positive regulatory relationship between PrPc and SATB1 may provide an explanation for the action of PrPc in CRC malignancy.

Although the biological function and clinical significance of SATB1 in cancers are well established, the detailed mechanism regulating SATB1 expression is still not clear. We showed here that SP1 is an important downstream target of PrPc explaining the mode of regulation of SATB1 expression at the molecular level in CRC cells. A direct and specific binding of SP1 to the SATB1 promoter was detected, which was largely abolished by $\mathrm{PrPc}$ depletion. PrPc is a membranous protein which functions as a receptor or co-receptor for extracellular matrix proteins such as laminin $(20,21)$ and vitronectin $(22)$, as well as the secreted co-chaperone stressinducible protein 1 (STI1) (23) to promote intracellular signaling-mediated downstream effetcs. In the present study, we demonstrated that the Fyn pathway lies downstream of PrPc. Inhibition of Fyn pathway abolished the molecular events downstream of PrPc, suggesting that the
Fyn pathway is required for PrPc-mediated SATB1 expression. Since inhibition of SP1 also reduced SATB1 expression and metastatic capacity in colorectal cancer cells, PrPc-Fyn-SP1SATB1 axis is a relevant molecular mechanism that links PrPc expression to poor prognosis in malignancies depending on a high rate of metastasis.

In conclusion, this study showed that PrPc expression in CRC confers a significant metastatic advantage for CRCs by a mechanism that involves the function of PrPc as an ancillary protein required for the function and expression of SATB1. Thus, PrPc could be a strong indicator for more aggressive tumors and possible poorer clinical outcome. Targeting pathways involved in metastasis such as PrPc-Fyn-HIF2 $\alpha$-SATB1 axis represents a promising avenue to inhibit maglinancy of colorectal cancer cells.

\section{References}

1. Jemal A, Siegel R, Ward E, Hao Y, Xu J, Murray T and Thun MJ: Cancer statistics. CA Cancer J Clin 58: 71-96, 2008.

2. Boyle P and Ferlay J: Cancer incidence and mortality in Europe, 2004. Ann Oncol 16: 481-488, 2005.

3. Bardelli A, Parsons DW, Silliman N, et al: Mutational analysis of the tyrosine kinome in colorectal cancers. Science 300: 949-950, 2003.

4. Stahl N, Borchelt DR, Hsiao K and Prusiner SB: Scrapie prion protein contains a phosphatidylinositol glycolipid. Cell 51: 229-249, 1987.

5. Brown DR, Qin K, Herms JW, et al: The cellular prion protein binds copper in vivo. Nature 390: 684-687, 1997.

6. Brown DR, Nicholas RS and Canevari L: Lack of prion protein expression results in a neuronal phenotype sensitive to stress. J Neurosci Res 67: 211-224, 2002.

7. Chen S, Mangé A, Dong L, Lehmann S and Schachner M: Prion protein as trans-interacting partner for neurons is involved in neurite outgrowth and neuronal survival. Mol Cell Neurosci 22: 227-233, 2003.

8. Kikuchi Y, Kakeya T, Yamazaki T, et al: G1-dependent prion protein expression in human glioblastoma cell line T98G. Biol Pharm Bull 25: 728-733, 2002.

9. Meslin F, Conforti R, Mazouni C, et al: Efficacy of adjuvant chemotherapy according to Prion protein expression in patients with estrogen receptor-negative breast cancer. Ann Oncol 18: 1793-1798, 2007.

10. Ochel HJ, Gademann G, Trepel J and Neckers L: Modulation of prion protein structural integrity by geldanamycin. Glycobiology 13: 655-660, 2003.

11. Pan Y, Zhao L, Liang J, et al: Cellular prion protein promotes invasion and metastasis of gastric cancer. FASEB J 20: 1886-1888, 2006.

12. Zhuang D, Liu Y, Mao Y, et al: TMZ-induced PrPc/par-4 interaction promotes the survival of human glioma cells. Int J Cancer 130: 309-318, 2012.

13. Liang J, Pan Y, Zhang D, et al: Cellular prion protein promotes proliferation and G1/S transition of human gastric cancer cells SGC7901 and AGS. FASEB J 21: 2247-2256, 2007.

14. Li QQ, Chen ZQ, Cao XX, et al: Involvement of NF- $\mathrm{kB} / \mathrm{miR}-448$ regulatory feedback loop in chemotherapy-induced epithelialmesenchymal transition of breast cancer cells. Cell Death Differ 18: 16-25, 2011

15. Boussiotis VA, Barber DL, Lee BJ, Gribben JG, Freeman GJ and Nadler LM: Differential association of protein tyrosine kinases with the T cell receptor is linked to the induction of anergy and its prevention by B7 family-mediated costimulation. J Exp Med 184: 365-376, 1996.

16. Natalwala A, Spychal R and Tselepis C: Epithelial-mesenchymal transition mediated tumourigenesis in the gastrointestinal tract. World J Gastroenterol 14: 3792-3797, 2008.

17. Du J, Pan Y, Shi Y, et al: Overexpression and significance of prion protein in gastric cancer and multidrug-resistant gastric carcinoma cell line SGC7901/ADR. Int J Cancer 113: 213-220, 2005.

18. Han HJ, Russo J, Kohwi Y and Kohwi-Shigematsu T: SATB1 reprogrammes gene expression to promote breast tumour growth and metastasis. Nature 452: 187-193, 2008. 
19. Kumar PP, Purbey PK, Sinha CK, Notani D, Limaye A, Jayani RS and Galande S: Phosphorylation of SATB1, a global gene regulator, acts as a molecular switch regulating its transcriptional activity in vivo. Mol Cell 22: 231-243, 2006.

20. Graner E, Mercadante AF, Zanata SM, et al: Cellular prion protein binds laminin and mediates neuritogenesis. Mol Brain Res 76: 85-92, 2000.

21. Graner E, Mercadante AF, Zanata SM, Martins VR, Jay DG and Brentani RR: Laminin-induced PC-12 cell differentiation is inhibited following laser inactivation of cellular prion protein. FEBS Lett 482: 257-260, 2000.
22. Hajj G N, Lopes MH, Mercadante AF, et al: Cellular prion protein interaction with vitronectin supports axonal growth and is compensated by integrins. J Cell Sci 120: 1915-1926, 2007.

23. Zanata SM, Lopes MH, Mercadante AF, et al: Stress-inducible protein 1 is a cell surface ligand for cellular prion that triggers neuroprotection. EMBO J 21: 3307-3316, 2002. 